\section{Avaliação dos indicadores de saúde bucal no Brasil: tendência evolutiva pró-equidade?}

\author{
Evaluation of oral health indicators in Brazil: a \\ trend towards equity in dental care?
}

\section{Evaluación de los indicadores de salud bucal en Brasil: ¿tendencia evolutiva pro-equidad?}

\section{Resumo}

Foi testada a hipótese de que os indicadores de saúde bucal, obtidos do Pacto da Atenção Básica de 2006, Pacto pela Saúde do biênio 2010/2011 e indicadores de transição entre o Pacto pela Saúde e Contrato Organizativo da Ação Pública em Saúde de 2012, não diferiam entre as Unidades Federativas (UF) brasileiras com diferentes Índices de Desenvolvimento Humano (IDH). Para isso, foi realizado um estudo longitudinal ecológico comparando as UF do Brasil com extremos de IDH. Os dados foram obtidos dos sistemas de informação do SUS e do Programa das Nações Unidas para o Desenvolvimento, e submetidos à análise de regressão linear de efeitos mistos (alfa $=5 \%$ ). Todos os indicadores de oportunidade de acesso foram associados negativamente com o IDH. Para os de utilização, a associação ocorreu para apenas dois indicadores. Os resultados evidenciaram uma tendência evolutiva pró-equidade para indicadores de oportunidade de acesso em relação à saúde bucal.

Desigualdades em Saúde; Iniquidade Social; Indicadores Básicos de Saúde; Saúde Bucal
Juliana de Kássia Braga Fernandes 1,2 Judith Rafaelle Oliveira Pinho 1 Rejane Christine de Sousa Queiroz 1 Erika Bárbara Abreu Fonseca Thomaz 1

\author{
1 Universidade Federal do \\ Maranhão, São Luís, Brasil. \\ 2 Universidade Ceuma, São \\ Correspondência \\ J. K. B. Fernandes \\ Rua Quefren 10, apto. 506, \\ São Luís, MA 65075-790, \\ Brasil. \\ juliana_kassia@hotmail.com
}




\section{Introdução}

O gradiente social como modulador da saúde bucal tem sido evidenciado em várias populações de diferentes países, independentemente da natureza, abrangência e eficiência dos respectivos sistemas de saúde 1,2. Estudos, tanto no nível individual 3,4 quanto ecológico 5,6, comprovam a relação entre as desigualdades sociais e a condição de saúde bucal, sugerindo a existência de iniquidades.

O Brasil é um país marcado por desigualdades socioeconômicas com potencial impacto na saúde 7,8 . Na tentativa de minimizar essas iniquidades, o Sistema Único de Saúde (SUS) prevê entre as suas diretrizes básicas o princípio da equidade ${ }^{9}$. Esse princípio tem orientado as políticas de saúde no Brasil desde a 8a Conferência Nacional de Saúde, intensificando-se a partir da edição da Norma Operacional Básica de 1996 (NOB/SUS 1996) 10, do Ministério da Saúde. Desde então, vários estudos têm observado uma tendência redistributiva da política de transferência de recursos financeiros do SUS por meio do Piso de Atenção Básica (PAB) 11, que tem favorecido municípios com baixo Índice de Desenvolvimento Humano (IDH) 12,13,14.

Para ampliar o monitoramento da aplicação dos recursos e do impacto na saúde da população, regulamentou-se, por meio da Portaria no 3.925 de 199815 e da Portaria no 476 de 1999 16, o Pacto de Indicadores da Atenção Básica (PIAB) 17, um instrumento formal de negociação nas três esferas de gestão (União, Estados e Municípios), com metas a serem alcançadas para indicadores previamente acordados, indicando a intenção de induzir melhorias na atenção básica e na saúde da população 18,19,20.

Em 2006, um conjunto de reformas institucionais do SUS foi pactuado, incorporando novas portarias e novos indicadores, os quais constituíram o Pacto pela Saúde (Pacto em Defesa do SUS; Pacto pela Vida e Pacto de Gestão) 21, que teve como objetivo promover inovações nos processos e instrumentos de gestão, redefinindo as responsabilidades de cada gestor em função das necessidades de saúde da população e na busca da equidade social 21,22.

Em 2011, foi agregado mais um instrumento de pactuação pelo Decreto no 7.508 , que regulamenta a Lei no 8.080, de 1990, instituindo o Contrato Organizativo da Ação Pública da Saúde (COAP) ${ }^{23}$, que objetiva a organização e a integração das ações e dos serviços, em regiões de saúde, com a finalidade de garantir a integralidade da assistência aos usuários 20,23. E, reconhecendo as iniquidades no acesso e utilização dos serviços de saúde no Brasil 24, publica-se também em 2011 a segunda edição da Política Nacional de Atenção Básica (PNAB) 25, incorporando o Programa Nacional de Melhoria do Acesso e da Qualidade da Atenção Básica (PMAQ-AB) 26 como uma estratégia de indução de melhorias no acesso e qualidade da Atenção Básica e ratificando a saúde bucal na agenda de prioridades do governo 27 .

Nesse contexto, os indicadores de saúde bucal constituem uma importante ferramenta para avalição dos recursos destinados a essa área na atenção básica e para avaliação do impacto da aplicação destes recursos na população 19,20. Esses indicadores devem, portanto, ser considerados na implementação de políticas públicas de forma equânime.

Poucos estudos utilizando indicadores de saúde para avaliar os serviços de saúde bucal da população têm levado em conta a condição socioeconômica 28,29,30. Segundo tais autores, foi possível encontrar indícios pró-equidade em alguns estados brasileiros. Nesses estudos, entretanto, foram incluídos apenas os indicadores previstos antes de 2006, não incluindo novas portarias e indicadores do Pacto pela Saúde 21 (Pacto em Defesa do SUS; Pacto pela Vida e Pacto de Gestão) e da transição Pacto pela Saúde/COAP 23. Além disso, os estudos são de caráter regional, e embora incidam sobre populações declaradas heterogêneas, não são capazes de refletir as diferentes regiões do país.

Dessa forma, o objetivo do presente trabalho foi avaliar se as associações entre a condição socioeconômica de diferentes unidades federativas (UF) do Brasil com os indicadores de saúde bucal ocorrem em favor da equidade, considerando a hipótese de que estados com as piores condições socioeconômicas apresentam melhores indicadores de saúde bucal.

\section{Métodos}

Realizou-se um estudo longitudinal ecológico, com dados de base populacional das seguintes UF: Distrito Federal, Santa Catarina, São Paulo, Rio de Janeiro, Paraíba, Piauí, Maranhão, Alagoas e Pará. Essas UF foram selecionadas de acordo com os valores do IDH, de forma a contemplar os estados pertencentes ao maior quintil (Distrito Federal, Santa Catarina, São Paulo e Rio de Janeiro, nos anos de 2000 e 2010) versus aqueles localizados no menor quintil de IDH (Paraíba, Piauí, Maranhão e Alagoas, em 2000, e Pará, Piauí, Maranhão e Alagoas, em 2010).

Para tratar o princípio da equidade foram consideradas como iniquidades as desigualdades injustas em diferentes condições 
socioeconômicas dos estados brasileiros. Dessa forma, o IDH foi o indicador utilizado para medir a condição socioeconômica 19,29, referente aos anos 2000 e 2010, obtido do Programa das Nações Unidas para o Desenvolvimento (PNUD) 31. O delineamento do trabalho foi assim proposto, incluindo UF com valores extremos de IDH, para que as iniquidades em saúde, caso existissem, pudessem ser evidenciadas nas análises.

As informações foram obtidas por meio de consultas aos bancos de dados do Ministério da Saúde (DATASUS. Informações em saúde. http://www2.datasus.gov.br/DATASUS/index. php?area=0201, acessado em 14/Jan/2015) e do PNUD 31. Os dados foram coletados em janeiro de 2015 e se referiram ao período de 2000 a 2012.

As variáveis de desfecho foram os indicadores de saúde bucal do PIAB de 2006 32, Pacto pela Saúde do biênio 2010/2011 21, e transição Pacto pela Saúde e COAP de 2012 23, obtidos no Sistema de Informação da Atenção Básica (SIAB) 33 nos sítios do Departamento de Informática do SUS (DATASUS. Informações em saúde. http:/ / www2.datasus.gov.br/DATASUS/index. php?area=0201, acessado em 14/Jan/2015). Esses indicadores constituem um instrumento nacional de monitoramento e avaliação das ações e serviços de saúde bucal.

Os indicadores de interesse em saúde bucal definidos pelo PIAB, de acordo com a Portaria GM/MS no 493 de 10 de março de 2006 32, incluídos neste estudo foram:

(1) Cobertura de primeira consulta odontológica programática: porcentagem de usuários que receberam a primeira consulta odontológica. Essa é realizada com o objetivo de diagnóstico e, essencialmente, elaboração de um plano preventivo-terapêutico, para atender às necessidades detectadas. Os atendimentos aleatórios como os de urgência/emergência que não têm previsão de continuidade não estão incluídos neste indicador.

(2) Cobertura da ação coletiva escovação dental supervisionada: percentual de cobertura referente à média de usuários que tiveram acesso à escovação dental com orientação/supervisão de um profissional treinado. É considerado o mês ou meses em que se realizou a atividade, o local e o ano, e esta deverá ser voltada à prevenção de doenças bucais, mais especificamente cárie dentária e doença periodontal. Essa ação é direcionada a um grupo de indivíduos, portanto, não inclui a atividade educativa individual.

(3) Média de procedimentos odontológicos básicos individuais: número médio de procedimentos odontológicos básicos, clínicos e/ou cirúrgicos realizados, por indivíduo, na população residente em determinado local e período.
(4) Proporção de procedimentos odontológicos especializados em relação às ações odontológicas individuais: proporção de procedimentos odontológicos especializados em relação às demais ações individuais odontológicas realizadas no âmbito do SUS.

(5) Proporção de população coberta pelo Programa Saúde da Família (PSF): número de pessoas cadastradas no PSF, de 2000 a 2007, por município, em relação à população desse período ${ }^{34}$. Embora esse indicador não se refira especificamente à saúde bucal, foi incluído como um importante indicador de acesso a serviços de saúde.

Os dados disponíveis no DATASUS para as variáveis Cobertura de primeira consulta odontológica programática e Cobertura da ação coletiva de escovação dental supervisionada, no período de coleta, são referentes aos anos de 2006 e 2007. Para as demais variáveis do PAB, os dados foram referentes ao período de 2000 a 2007.

Entre os indicadores do Pacto pela Saúde 21,22, incluíram-se:

(1) Média da ação coletiva de escovação dental supervisionada, indicador que reflete, em percentual, o número médio de pessoas que participaram da ação coletiva escovação dental supervisionada. Por escovação dental supervisionada entende-se a escovação com ou sem evidenciação de placas bacterianas, realizada com grupos populacionais sob orientação e supervisão de um ou mais profissionais de saúde. A ação é registrada por usuário por mês, independentemente da frequência com que é realizada (diária, semanal, quinzenal, mensal, ou duas, três ou quatro vezes por ano) ou da frequência com que o usuário participou da ação 35 . Os dados dessa variável foram referentes ao período de 2008 a 2011.

(2) Cobertura populacional estimada das equipes de saúde bucal (ESB) da Estratégia Saúde da Família (ESF): representa o número de ESB da ESF implantadas (modalidades I e II) x 3.450 pessoas em relação à população no mesmo local e período 36 .

(3) Proporção da população cadastrada pela ESF: população cadastrada no SIAB em determinado local e período em relação à população definida pelo censo em cada ano 37 .

Tanto para a Proporção da população cadastrada pela ESF quanto para a Cobertura populacional estimada das ESB da ESF, os dados foram referentes ao período de 2007 a 2011.

Por fim, foram três os indicadores de saúde bucal definidos pela transição entre o Pacto pela Saúde e COAP, cujos dados estão disponíveis no período de 2008 a 2012 23,37. São eles:

(1) Média da ação coletiva de escovação dental supervisionada: número de pessoas 
participantes na ação coletiva de escovação dental supervisionada realizada em determinado local em 12 meses $\div 12$, dividido pela população no mesmo local e período.

(2) Cobertura populacional estimada pelas ESB: soma da carga horária dos cirurgiões-dentistas/40, multiplicada por 3.000, dividida pela população no mesmo local e período.

(3) Cobertura populacional estimada pelas equipes de atenção básica: número de equipe ESF somado ao número de equipe ESF equivalente, multiplicado por 3.000 , dividido pela população no mesmo local e período.

Segundo Viana et al. 38, para análise e elaboração de políticas públicas a aplicação do conceito de equidade deve ocorrer em três vertentes: distribuição dos recursos, oferta ou oportunidade de acesso e utilização dos serviços. Neste estudo, as variáveis de desfecho foram analisadas, segundo sua própria definição, em variáveis de oportunidade de acesso e em variáveis de utilização dos serviços. Assim, foram consideradas como variáveis de oportunidade de acesso: Proporção da população coberta pelo PSF (PIAB), Cobertura populacional estimada das ESB da ESF (Pacto pela Saúde), Proporção da população cadastrada pela ESF (Pacto pela Saúde), Cobertura populacional estimada pelas ESB (COAP) e Cobertura populacional estimada pelas equipes de atenção básica (COAP). As demais foram analisadas como variáveis de utilização dos serviços.

Para a avaliação da equidade, foi realizada análise bivariada por meio da correlação de Spearman (Rs), considerando como variável explicativa o IDH e como variáveis de desfecho os indicadores selecionados do PIAB 32, Pacto pela Saúde 21 e transição Pacto pela Saúde/COAP 23. Foram aceitas como correlações a favor da equidade aquelas que foram significativas e inversamente correlacionadas.

Por se tratar de estudo em que os dados longitudinais apresentam estrutura hierárquica (medidas repetidas para a mesma UF), optou-se por um tipo de análise de efeitos mistos - modelo de intercepto randômico - no qual o coeficiente é fixo, mas o intecepto é randômico ${ }^{39}$. Nessa análise, a porção randômica é considerada sem a inclusão de variáveis. Ou seja, como modelo vazio. Isso permite a incorporação do efeito do intercepto randômico na estrutura de análise 40 . Essa modelagem permite analisar dados longitudinais desbalanceados (medidas obtidas em cada UF observadas em tempos diferentes) em estrutura hierárquica, incorporando a dependência e a matriz de variância e covariância das unidades 40 .

Considerou-se o nível de significância de $5 \%$. As análises foram realizadas com o software
Stata, versão 11.0 (StataCorp LP, College Station, Estados Unidos).

\section{Resultados}

O ranking das UF com o maior quintil de IDH não variou entre 2000 e 2010, permanecendo Distrito Federal, Santa Catarina, São Paulo e Rio de Janeiro. Porém, houve variação no ranking das UF com o menor quintil de IDH: em 2010, substituiu-se a Paraíba pelo Pará, e as demais UF permaneceram constantes. O IDH médio das UF do lo quintil foi 0,484 (em 2000) e 0,640 (em 2010), inferiores às médias nacionais dos períodos $(0,576$ e 0,704$)$. Em contrapartida, as UF do 5o quintil obtiveram médias $20 \%$ (2000) e $11 \%$ (2010) superiores às nacionais 31 . Dessa forma, têm-se grupos de UF com indicadores extremos de desigualdade socioeconômica.

Na Tabela 1 são apresentadas as análises descritivas dos indicadores de saúde bucal do PIAB 32 Pacto pela Saúde 21 e de transição Pacto pela Saúde/COAP 23 para as UF de menor (1o quintil) e maior IDH (5o quintil), de acordo com a classificação de 2000 e 2010. Para todos os indicadores de acesso, as médias e medianas das UF pertencentes ao quinto quintil de IDH apresentaram valores menores que as do primeiro quintil.

Entre os indicadores de acesso, observouse correlação negativa $(\mathrm{p}<0,001)$ do IDH com: Proporção de população coberta pelo PSF ( $\mathrm{R}=$ -0,65, em 2000; $\mathrm{R}=-0,56$, em 2010); Cobertura populacional estimada das ESB da ESF $(R=-0,67$, em 2000; $R=$-0,62, em 2010); Proporção da população cadastrada pela ESF $(R=-0,68$, em 2000; $R=$ -0,64, em 2010); Cobertura populacional estimada pelas ESB ( $R=-0,65$, em 2000; $R=-0,52$, em 2010); e Cobertura populacional estimada pelas equipes de atenção básica $(R=-0,72$, em 2000; $R=$ -0,57, em 2010).

Entre os indicadores de utilização, a Cobertura de primeira consulta odontológica programática $(R=-0,65$, em 2000; $R=-0,66$, em 2010) apresentou correlação negativa com o IDH ( $\mathrm{p}=$ 0,006) e Proporção de procedimentos odontológicos especializados em relação às ações odontológicas individuais $(R=0,71$, em 2000; $R=0,45$ em 2010), correlação positiva $(\mathrm{p}<0,001)$.

Portanto, todos os indicadores de acesso mostraram correlação negativa com o IDH; o mesmo não ocorreu com os de utilização de serviços, em que apenas um apresentou correlação negativa e outro, positiva.

$\mathrm{Na}$ análise de regressão multivariada ajustada, verificou-se que as UF pertencentes ao maior quintil de IDH apresentaram os menores valores para todos os indicadores de acesso para as UF 
Estatística descritiva das variáveis de saúde bucal do Pacto de Indicadores da Atenção Básica (PIAB), Pacto pela Saúde e transição Pacto pela Saúde e Contrato Organizativo da Ação Pública da Saúde (COAP) para as Unidades Federativas do 1o e 5o quintis do Índice de Desenvolvimento Humano (IDH)

\begin{tabular}{|c|c|c|c|c|c|c|c|c|c|c|c|}
\hline Indicador & Quintil & $n^{*}$ & Ano ** & Média & DP & CV & Mínimo & $P_{25}$ & Mediana & $P_{75}$ & Máximo \\
\hline \multicolumn{12}{|l|}{ PIAB } \\
\hline Cobertura de primeira & 1으 & 2 & 2000 & 15,68 & 1,92 & 12,24 & 13,04 & 14,16 & 15,87 & 17,09 & 18,18 \\
\hline consulta odontológica & 5으 & & $2000 / 2010$ & 10,84 & 3,90 & 35,98 & 6,42 & 8,42 & 9,89 & 13,45 & 16,79 \\
\hline programática (utilização) & 1으 & & 2010 & 15,37 & 2,04 & 13,27 & 13,04 & 13,59 & 15,17 & 17,09 & 18,18 \\
\hline Cobertura da ação & 1으 & 2 & 2000 & 1,68 & 1,20 & 71,43 & 0,93 & 0,97 & 1,08 & 2,16 & 4,11 \\
\hline coletiva escovação dental & 5으 & & $2000 / 2010$ & 2,06 & 1,78 & 86,41 & 0,09 & 0,40 & 2,12 & 3,73 & 3,90 \\
\hline supervisionada (utilização) & 1 으 & & 2010 & 1,56 & 1,29 & 82,69 & 0,46 & 0,83 & 0,97 & 2,16 & 4,11 \\
\hline Média de procedimentos & 1으 & 8 & 2000 & 0,54 & 0,08 & 14,81 & 0,35 & 0,49 & 0,53 & 0,59 & 0,74 \\
\hline odontológicos básicos & 5으 & & $2000 / 2010$ & 0,52 & 0,17 & 32,69 & 0,25 & 0,41 & 0,52 & 0,63 & 0,92 \\
\hline individuais (utilização) & 1으 & & 2010 & 0,53 & 0,08 & 15,09 & 0,35 & 0,48 & 0,54 & 0,58 & 0,68 \\
\hline Proporção de procedimentos & 1으 & 8 & 2000 & 5,56 & 1,37 & 24,64 & 2,77 & 4,49 & 5,30 & 6,32 & 8,36 \\
\hline odontológicos & 5으 & & $2000 / 2010$ & 8,45 & 1,79 & 21,18 & 4,63 & 7,31 & 8,21 & 9,76 & 11,93 \\
\hline especializados em relação & 1으 & & 2010 & 6,80 & 3,24 & 47,65 & 2,77 & 4,56 & 5,47 & 8,59 & 14,71 \\
\hline \multicolumn{12}{|l|}{ às ações odontológicas } \\
\hline \multicolumn{12}{|l|}{ individuais (utilização) } \\
\hline Proporção da população & 1으 & 8 & 2000 & 59,47 & 21,79 & 36,64 & 7,17 & 45,09 & 66,00 & 75,28 & 90,81 \\
\hline \multirow[t]{2}{*}{ coberta pelo PSF (acesso) } & 5으 & & $2000 / 2010$ & 21,92 & 18,61 & 84,90 & 3,84 & 7,87 & 16,57 & 25,08 & 66,38 \\
\hline & 1으 & & 2010 & 48,83 & 25,41 & 52,04 & 6,03 & 24,31 & 54,86 & 69,39 & 90,81 \\
\hline \multicolumn{12}{|l|}{ Pacto pela Saúde } \\
\hline Média da ação coletiva & 1으 & 4 & 2000 & 2,36 & 0,97 & 41,10 & 1,15 & 1,52 & 2,11 & 3,14 & 4,30 \\
\hline de escovação dental & 5으 & & $2000 / 2010$ & 1,99 & 1,43 & 71,86 & 0,11 & 0,38 & 0,69 & 0,96 & 4,44 \\
\hline supervisionada (utilização) & 1으 & & 2010 & 2,01 & 1,20 & 59,70 & 0,41 & 1,09 & 1,76 & 2,93 & 4,30 \\
\hline Cobertura populacional & 1으 & 5 & 2000 & 81,75 & 21,59 & 26,41 & 55,58 & 62,17 & 79,87 & 102,31 & 108,64 \\
\hline estimada das ESB da ESF & 5으 & & $2000 / 2010$ & 17,00 & 15,32 & 90,12 & 0,27 & 5,84 & 12,18 & 26,79 & 46,38 \\
\hline (acesso) & 1으 & & 2010 & 60,65 & 30,06 & 49,56 & 12,66 & 40,05 & 62,17 & 79,87 & 108,64 \\
\hline Proporção da população & 1으 & 5 & 2000 & 83,25 & 9,60 & 11,53 & 68,61 & 74,48 & 84,80 & 92,45 & 95,72 \\
\hline \multirow[t]{2}{*}{ cadastrada pela ESF (acesso) } & 5으 & & $2000 / 2010$ & 33,09 & 22,46 & 67,88 & 8,48 & 17,96 & 25,90 & 48,28 & 71,76 \\
\hline & 1으 & & 2010 & 70,08 & 20,85 & 29,75 & 33,29 & 56,73 & 74,48 & 86,38 & 95,72 \\
\hline \multicolumn{12}{|l|}{ Transição Pacto pela Saúde/ } \\
\hline \multicolumn{12}{|l|}{ COAP } \\
\hline Média da ação coletiva & 1으 & 5 & 2000 & 2,30 & 0,92 & 40,00 & 1,15 & 1,53 & 2,11 & 3,07 & 4,30 \\
\hline de escovação dental & 5으 & & $2000 / 2010$ & 2,02 & 1,39 & 68,81 & 0,11 & 0,39 & 2,68 & 2,95 & 4,42 \\
\hline supervisionada (utilização) & 1으 & & 2010 & 1,97 & 1,16 & 58,88 & 0,38 & 1,09 & 1,77 & 2,93 & 4,30 \\
\hline Cobertura populacional & 1으 & 5 & 2000 & 72,81 & 14,27 & 19,60 & 53,74 & 59,44 & 71,33 & 88,29 & 90,30 \\
\hline \multirow[t]{2}{*}{ estimada pelas ESB (acesso) } & 5으 & & $2000 / 2010$ & 32,65 & 14,93 & 45,73 & 13,46 & 23,21 & 30,48 & 43,43 & 57,35 \\
\hline & 1으 & & 2010 & 57,91 & 19,68 & 33,98 & 25,76 & 43,26 & 59,45 & 71,33 & 88,32 \\
\hline Cobertura populacional & 1으 & 5 & 2000 & 83,12 & 9,78 & 11,77 & 71,32 & 72,74 & 84,59 & 92,86 & 93,80 \\
\hline estimada pelas equipes de & 5으 & & $2000 / 2010$ & 46,29 & 15,39 & 33,25 & 27,30 & 35,46 & 40,73 & 59,96 & 70,95 \\
\hline atenção básica (acesso) & 1으 & & 2010 & 70,40 & 19,28 & 27,39 & 38,25 & 56,87 & 72,74 & 85,22 & 93,80 \\
\hline
\end{tabular}

CV: coeficiente de variação; DP: desvio padrão; ESB: equipes de saúde bucal; ESF: Estratégia Saúde da Família; $P_{25}$ : percentil 25; $P_{75}$ : percentil 75; PSF: Programa Saúde da Família.

* Número de anos analisados;

** Ano de estimativa do IDH. 
selecionadas pelo IDH 2000 ( $\mathrm{p}<0,001)$. Para as unidades selecionadas pelo IDH 2010, a associação negativa ocorreu para a Cobertura populacional estimada das ESB da ESF $(\beta=-10,91 ; p=$ $0,02)$ e Proporção da população cadastrada pela ESF $(\beta=-9,25 ; p=0,03$ ) (Figura 1, Tabela 2). O mesmo foi observado para o indicador de utilização Cobertura de primeira consulta odontológica programática $(\beta=-1,21 ; \mathrm{p}=0,03$, em 2000; e $\beta=$ $-1,13 ; p=0,04$, em 2010). No entanto, a Proporção de procedimentos odontológicos especializados em relação às ações odontológicas individuais foi maior entre as UF pertencentes ao quintil de maior IDH, e apenas para as UF selecionadas pelo IDH $2000(\beta=0,72 ; p=0,001)$ (Figura 2, Tabela 3).

\section{Discussão}

De acordo com os dados deste estudo, foi possível observar associação entre indicadores de saúde bucal, dos últimos instrumentos de pactuação, com o IDH, especialmente em relação aos indicadores relacionados com a oportunidade de acesso. A hipótese nula de que indicadores de saúde bucal não diferem entre as UF brasileiras com diferentes condições socioeconômicas foi rejeitada. Os indicadores de oportunidade de acesso foram melhores nos estados com menor IDH, sugerindo que a tendência próequidade observada no nível regional em estudos anteriores 29,30 também esteja ocorrendo em escala nacional.

Figura 1

Evolução dos indicadores de acesso, segundo grupos de Unidade da Federação (UF) com menor (1o quintil) e maior (5o quintil) Índice de Desenvolvimento Humano (IDH)

1a) Proporção da população coberta pelo PSF (PIAB), segundo grupos de UF do 1 o quintil e 5 o quintil selecionadas pelo IDH de 2000. Brasil, 2000-2007 *,**

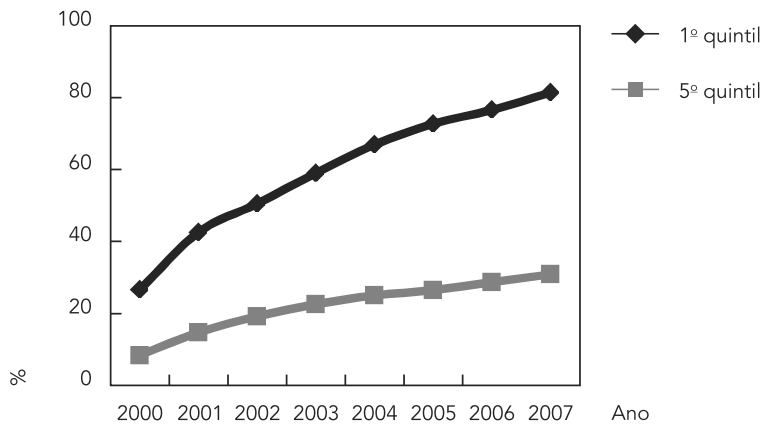

1c) Cobertura populacional estimada das ESB da ESF (Pacto pela Saúde), segundo grupos de UF do 1ㅇ quintil e 5ㅇ quintil selecionadas pelo IDH de 2000. Brasil, 2007-2011*,**

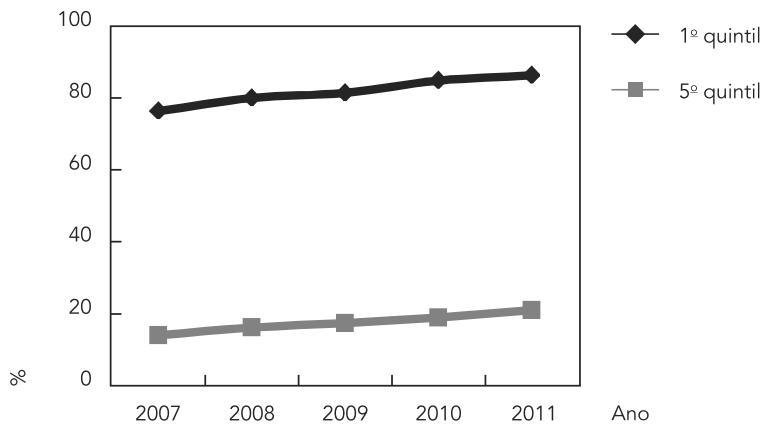

1b) Proporção da população coberta pelo PSF (PIAB), segundo grupos de UF do 1ㅇ quintil e 5 o quintil selecionadas pelo IDH de 2010. Brasil, 2000-2007 **

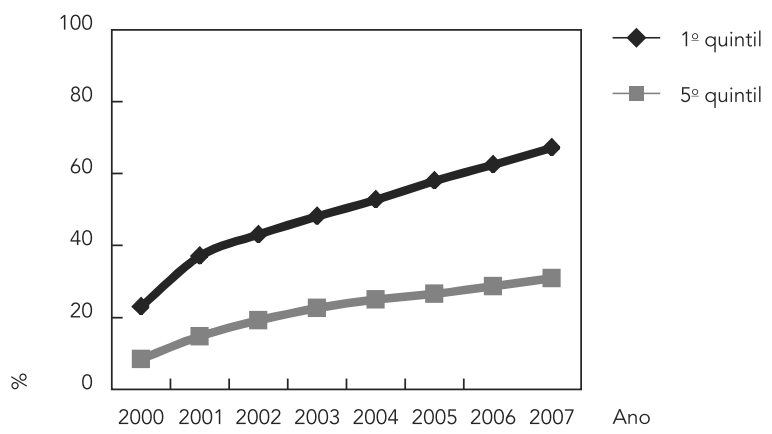

1d) Cobertura populacional estimada das ESB da ESF (Pacto pela Saúde), segundo grupos de UF do 1ㅇquintil e 5 ㅇ quintil selecionadas pelo IDH de 2010. Brasil, 2007-2011 *,**

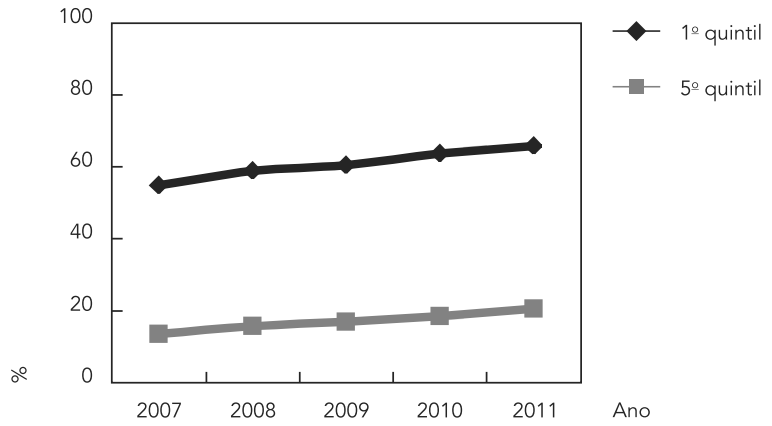


Evolução dos indicadores de acesso, segundo grupos de Unidade da Federação (UF) com menor (1o quintil) e maior (5o quintil) Índice de Desenvolvimento Humano (IDH).

1e) Proporção da população cadastrada pela ESF (Pacto pela Saúde), segundo grupos de UF do 1ㅇ quintil e 5o quintil selecionadas pelo IDH de 2000. Brasil, 2007-2011 *

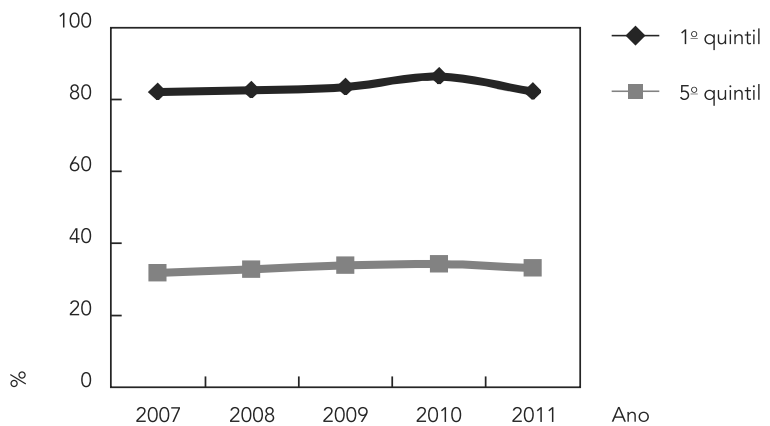

1g) Cobertura populacional estimada pelas ESB (Pacto pela Saúde/ COAP), segundo grupos de UF do 1o quintil e 5o quintil selecionadas pelo IDH de 2000. Brasil, 2008-2012 *,**

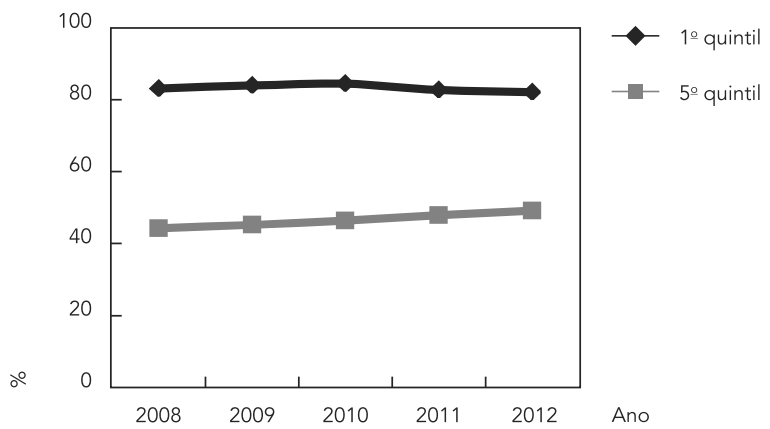

1f) Proporção da população cadastrada pela ESF (Pacto pela Saúde), segundo grupos de UF do 1ㅇ quintil e 5o quintil selecionadas pelo IDH de 2000. Brasil, 2007-2011 *

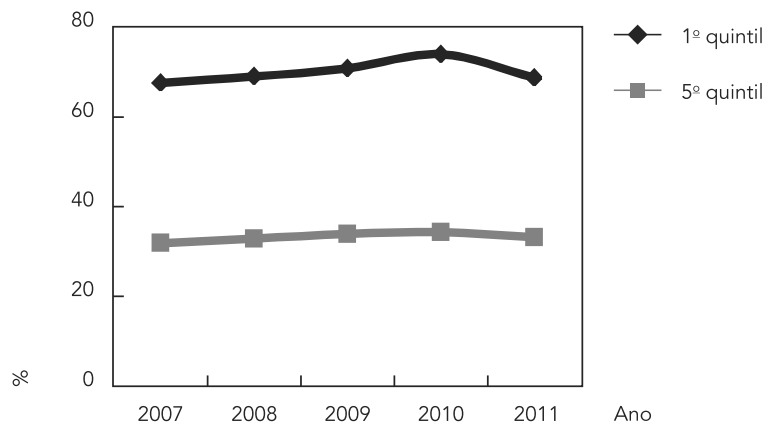

1h) Cobertura populacional estimada pelas ESB (Pacto pela Saúde/ COAP), segundo grupos de UF do 1ㅇ quintil e 5o quintil selecionadas pelo IDH de 2010. Brasil, 2008-2012 **

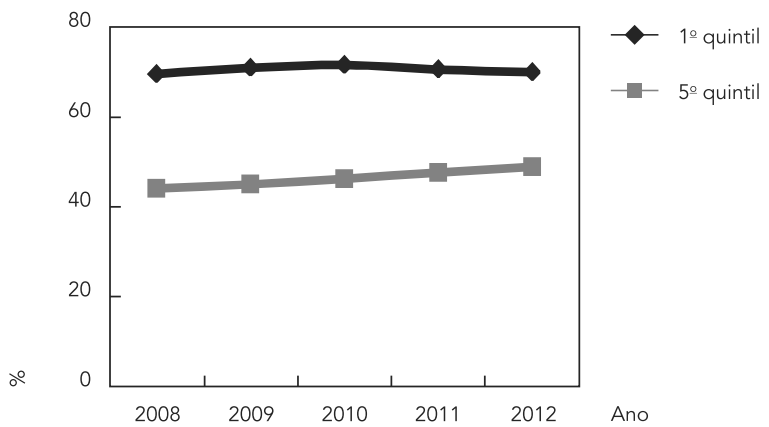

(continua)

A condição socioeconômica, no entanto, não deve ser interpretada como a causa para o melhor ou pior desempenho dos indicadores de saúde analisados, o que favoreceria a hipótese da existência de equidade nas políticas de saúde pública no Brasil. O que pode ser considerado na interpretação dos dados é a "tendência de equidade" quando as associações ocorrem a favor dos estados com desvantagens socioeconômicas. Esse termo vem sendo adotado por estudos com metodologias semelhantes 19,29. Neste trabalho, a tendência pró-equidade observada foi baseada na associação negativa dos indicadores de saú- de relacionados com a oportunidade de acesso com o IDH.

É possível a interpretação cautelosa de que a PNAB vem sendo bem-sucedida em favorecer o acesso para as UF que apresentam maiores desvantagens socioeconômicas e, por seguinte, maiores necessidades em saúde 28,41, em consonância com o princípio de equidade da legislação do SUS. 
Evolução dos indicadores de acesso, segundo grupos de Unidade da Federação (UF) com menor (1ㅇ quintil) e maior (5o quintil) Índice de Desenvolvimento Humano (IDH).

1i) Cobertura populacional estimada pelas equipes de atenção básica (Pacto pela Saúde/COAP), segundo grupos de UF do 1ㅇ quintil e 5o quintil selecionadas pelo IDH de 2000. Brasil, 2008-2012 *,**

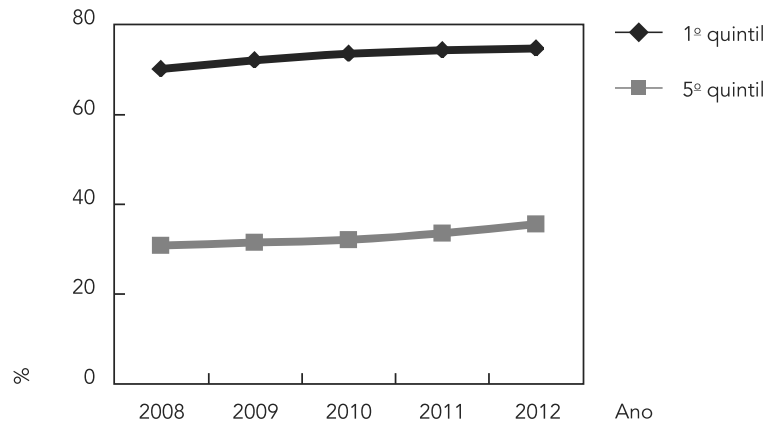

1j) Cobertura populacional estimada pelas equipes de atenção básica (Pacto pela Saúde/COAP), segundo grupos de UF do 1ㅇ quintil e 5으 quintil selecionadas pelo IDH de 2010. Brasil, 2008-2012

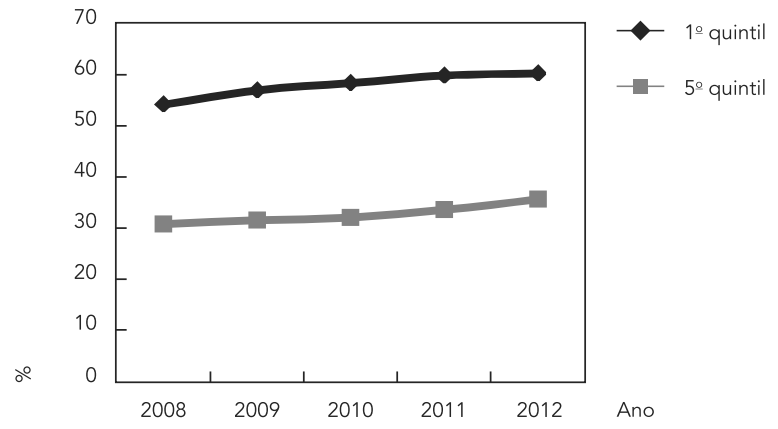

COAP: Contrato Organizativo da Ação Pública de Saúde; ESB: equipes de Saúde Bucal; ESF: Estratégia Saúde da Família; PIAB: Pacto de Indicadores da Atenção Básica; PSF: Programa Saúde da Família.

* Diferença estatisticamente significante entre os quintis;

** Diferença estatisticamente significante entre os anos.

\section{Indicadores de acesso}

$\mathrm{Na}$ análise de associação, todos os indicadores relacionados com a oportunidade de acesso à Atenção Básica foram associados negativamente com a condição socioeconômica para as UF selecionadas a partir do IDH 2000. Para as UF selecionadas pelo IDH 2010, essa associação ocorre para os indicadores Cobertura populacional estimada das ESB da ESF e a Proporção da população cadastrada pela ESF.

A diferença entre os resultados das UF selecionadas pelo IDH 2000 e IDH 2010 se dá pela substituição da Paraíba pelo Pará. Assim, as peculiaridades do Pará devem ser consideradas na interpretação dos resultados. Trata-se do segundo maior estado brasileiro, inserido quase que em totalidade na Região Amazônica e com uma bacia hidrográfica que abrange uma área de $1.253 .164 \mathrm{~km}^{2}$. As características geográficas dessa UF podem implicar dificuldades de acesso às unidades básicas de saúde em algumas regiões do estado, estabelecendo o hábito cultural da população pela procura espontânea pelo serviço de referência quaternária, hospitalar e de mais fácil acesso, levando a uma baixa cobertura na atenção primária 30 em relação à Paraíba.
Para o indicador Proporção da população coberta pelo PSF observou-se uma tendência evolutiva da coberta pelo PSF, com medianas maiores para os estados com a pior condição socioeconômica. De fato, de 1998 a 2004 houve uma importante expansão da cobertura do PSF/ESF, privilegiando os municípios de menor IDH. Já em 2004, a proporção da cobertura do PSF no Nordeste já chegava a $55 \%$ contra $38 \%$ na Região Sul ${ }^{42}$. Essa expansão parece que ocorre de forma continuada, segundo os dados do presente estudo.

A Proporção da população cadastrada pela ESF e Cobertura populacional estimada pelas equipes de atenção básica apresentaram tendência a favor da equidade. Considerando que as estimativas para esses indicadores foram feita a partir de 2007, pode-se especular que o incentivo para a expansão da cobertura da atenção básica, ocorrido na última década em todo o país 42 , tenha amenizado as diferenças entre os grupos de estados pesquisados; reforçando a conjectura de que existe uma expansão continuada da cobertura na atenção básica, que vem privilegiando os estados com menor IDH, diminuindo as diferenças entre os estados com diferentes condições socioeconômicas. 
Análise multivariada de regressão linear de efeitos mistos (modelagem intercepto randômico) entre indicadores de acesso e diferentes quintis do Índice de Desenvolvimento Humano (IDH).

\begin{tabular}{|c|c|c|c|c|c|c|c|c|}
\hline & \multicolumn{4}{|c|}{ IDH 2000} & \multicolumn{4}{|c|}{ IDH 2010} \\
\hline & $\beta$ & DP & IC95\% & $\begin{array}{l}\text { Valor } \\
\text { de p }\end{array}$ & $\beta$ & DP & $\mathrm{IC} 95 \%$ & $\begin{array}{l}\text { Valor } \\
\text { de } p\end{array}$ \\
\hline \multicolumn{9}{|c|}{ Proporção da população coberta pelo PSF (PIAB) } \\
\hline \multicolumn{9}{|l|}{ Efeito fixo } \\
\hline Intercepto & 50,53 & 10,08 & 30,$77 ; 70,30$ & $<0,001$ & 40,2 & 13,39 & 13,$94 ; 66,45$ & 0,003 \\
\hline Quintil & $-9,37$ & 2,75 & $-14,9 ;-3,99$ & 0,001 & $-6,73$ & 3,69 & $-13,96 ; 0,51$ & 0,07 \\
\hline Ano & 5,23 & 0,50 & 4,$26 ; 6,21$ & $<0,001$ & 4,39 & 0,42 & 3,$55 ; 5,22$ & $<0,001$ \\
\hline \multicolumn{9}{|l|}{ Efeito aleatório } \\
\hline Intercepto & & 15,24 & & & & 20,70 & & \\
\hline Resíduo & & 9,13 & & & & 7,80 & & \\
\hline \multicolumn{9}{|c|}{ Cobertura populacional estimada das ESB da ESF } \\
\hline \multicolumn{9}{|c|}{ (Pacto pela Saúde) } \\
\hline \multicolumn{9}{|l|}{ Efeito fixo } \\
\hline Intercepto & 79,04 & 13,30 & 52,$96 ; 105,12$ & $<0,001$ & 51,83 & 17,01 & 18,$51 ; 85,16$ & 0,002 \\
\hline Quintil & $-16,19$ & 3,66 & $-23,37 ;-9,001$ & $<0,001$ & $-10,91$ & 4,69 & $-20,12 ;-1,71$ & 0,02 \\
\hline Ano & 2,09 & 1,17 & 1,$75 ; 2,44$ & $<0,001$ & 2,19 & 0,18 & 1,$84 ; 2,54$ & $<0,001$ \\
\hline \multicolumn{9}{|l|}{ Efeito aleatório } \\
\hline Intercepto & & 20,71 & & & & 26,55 & & \\
\hline Resíduo & & 1,57 & & & & 1,60 & & \\
\hline \multicolumn{9}{|c|}{ Proporção da população cadastrada pela ESF } \\
\hline \multicolumn{9}{|l|}{ (Pacto pela Saúde) } \\
\hline \multicolumn{9}{|l|}{ Efeito fixo } \\
\hline Intercepto & 92,10 & 12,48 & 67,$62 ; 116,57$ & $<0,001$ & 74,16 & 15,65 & 43,$49 ; 104,83$ & $<0,001$ \\
\hline Quintil & $-12,54$ & 3,41 & $-19,23 ;-5,84$ & $<0,001$ & $-9,25$ & 4,28 & $-17,64 ;-0,85$ & 0,03 \\
\hline Ano & 0,41 & 0,23 & $-0,04 ; 0,86$ & 0,07 & 0,57 & 0,28 & 0,$03 ; 1,12$ & 0,04 \\
\hline \multicolumn{9}{|l|}{ Efeito aleatório } \\
\hline Intercepto & & 19,28 & & & & 24,21 & & \\
\hline Resíduo & & 2,08 & & & & 2,49 & & \\
\hline \multicolumn{9}{|c|}{ Cobertura populacional estimada pelas ESB (Pacto } \\
\hline \multicolumn{9}{|c|}{ pela Saúde/COAP) } \\
\hline \multicolumn{9}{|l|}{ Efeito fixo } \\
\hline Intercepto & 71,29 & 10,53 & 50,$65 ; 91,93$ & $<0,001$ & 50,79 & 12,55 & 26,$20 ; 75,38$ & $<0,001$ \\
\hline Quintil & $-10,04$ & 2,87 & $-15,66 ;-4,42$ & $<0,001$ & $-6,31$ & 3,44 & $-13,05 ; 0,43$ & 0,06 \\
\hline Ano & 1,16 & 1,20 & 0,$77 ; 1,54$ & $<0,001$ & 1,34 & 1,20 & 0,$97 ; 1,71$ & $<0,001$ \\
\hline \multicolumn{9}{|c|}{ Efeito aleatório } \\
\hline Intercepto & & 16,21 & & & & 19,45 & & \\
\hline Resíduo & & 1,77 & & & & 1,70 & & \\
\hline \multirow{2}{*}{\multicolumn{9}{|c|}{$\begin{array}{l}\text { Cobertura populacional estimada pelas equipes de } \\
\text { atenção básica (Pacto pela Saúde/COAP) }\end{array}$}} \\
\hline & & & & & & & & \\
\hline \multicolumn{9}{|l|}{ Efeito fixo } \\
\hline Intercepto & 87,88 & 9,42 & 69,$41 ; 106,35$ & $<0,001$ & 70,05 & 12,62 & 45,$31 ; 94,70$ & $<0,001$ \\
\hline Quintil & $-9,21$ & 2,54 & $-14,18 ;-4,24$ & $<0,001$ & $-0,03$ & 3,45 & $-12,79 ; 0,73$ & 0,08 \\
\hline Ano & 0,44 & 0,23 & 0,$001 ; 0,89$ & 0,05 & 0,64 & 0,22 & 0,$21 ; 1,06$ & 0,003 \\
\hline \multicolumn{9}{|l|}{ Efeito aleatório } \\
\hline Intercepto & & 14,32 & & & & 19,49 & & \\
\hline Resíduo & & 2,11 & & & & 2,01 & & \\
\hline
\end{tabular}

$\beta$ : coeficiente de regressão; COAP: Contrato Organizativo da Ação Pública de Saúde; DP: desvio padrão; ESB: equipes de saúde bucal; ESF: Estratégia Saúde da Família; IC95\%: intervalo de 95\% de confiança; PIAB: Pacto de Indicadores da Atenção Básica; PSF: Programa Saúde da Família. 
Figura 2

Evolução dos indicadores de utilização, segundo grupos de Unidade da Federação (UF) com menor (1ㅇ quintil) e maior (5o quintil) Índice de Desenvolvimento Humano (IDH).

2a) Cobertura de primeira consulta odontológica programática (PIAB), segundo grupos de UF do 1o quintil e 5o quintil selecionadas pelo IDH de 2000. Brasil, 2006-2007 *

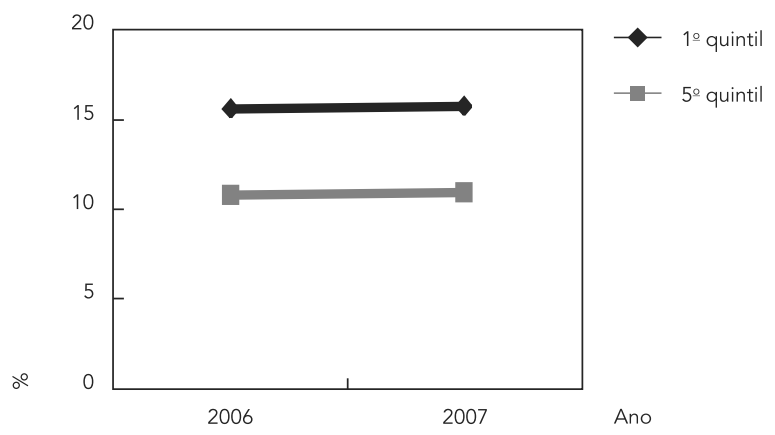

2c) Cobertura da ação coletiva escovação dental supervisionada (PIAB), segundo grupos de UF do 1ㅇq quintil e 5 ㅇ quintil selecionadas pelo IDH de 2000. Brasil, 2006-2007

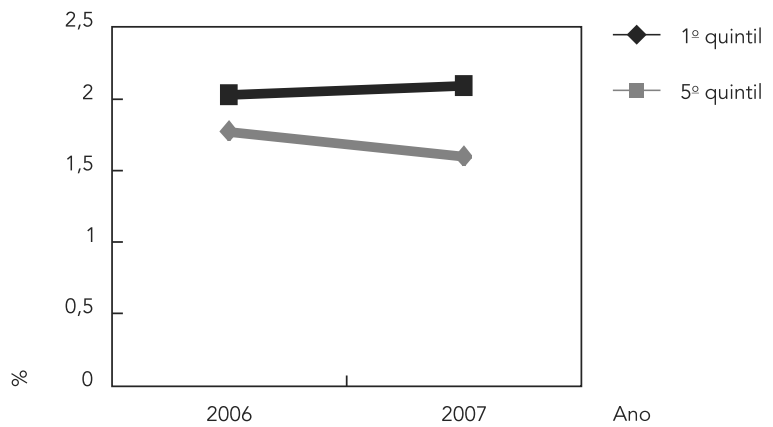

2e) Média de procedimentos odontológicos básicos individuais (PIAB), segundo grupos de UF do 1o quintil e 5 o quintil selecionadas pelo IDH de 2000. Brasil, 2000-2007 **

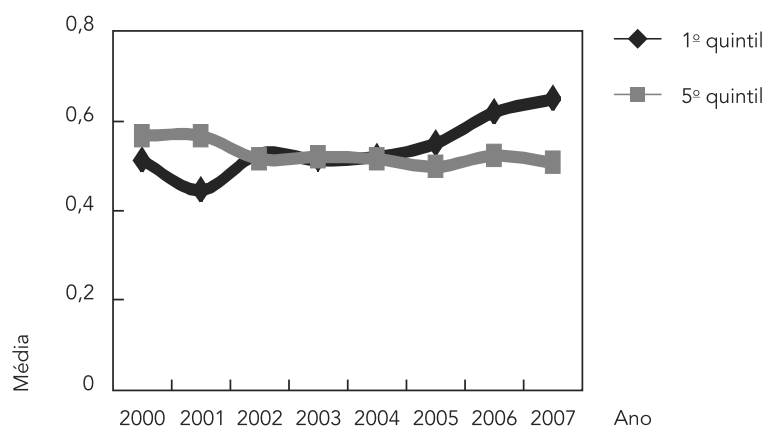

2b) Cobertura de primeira consulta odontológica programática (PIAB), segundo grupos de UF do 1ㅇ quintil e 5o quintil selecionadas pelo IDH de 2010. Brasil, 2006-2007 *

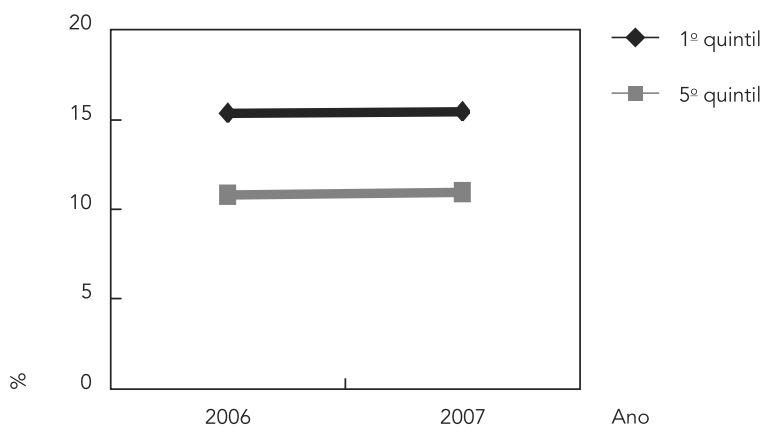

2d) Cobertura da ação coletiva escovação dental supervisionada (PIAB), segundo grupos de UF do 1ㅇ quintil e 5 ㅇ quintil selecionadas pelo IDH de 2010. Brasil, 2006-2007

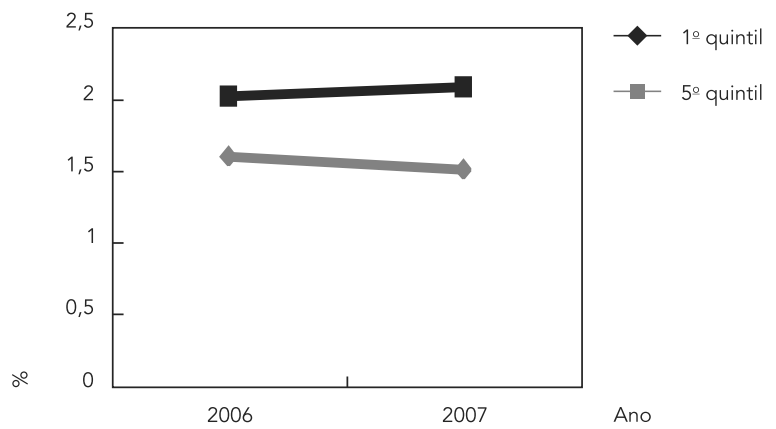

2f) Média de procedimentos odontológicos básicos individuais (PIAB), segundo grupos de UF do 1o quintil e 5o quintil selecionadas pelo IDH de 2010. Brasil, 2000-2007

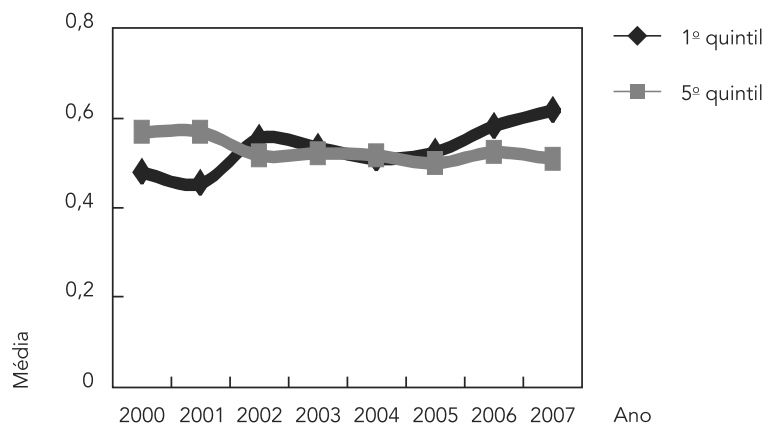

(continua) 
Evolução dos indicadores de utilização, segundo grupos de Unidade da Federação (UF) com menor (1ㅇquintil) e maior (5o quintil) Índice de Desenvolvimento Humano (IDH).

2g) Proporção de procedimentos odontológicos especializados em relação às ações odontológicas individuais (PIAB), segundo grupos de UF do 1 ㅇ quintil e 5 ㅇ quintil selecionadas pelo IDH de 2000. Brasil, 2000-2007 *,**

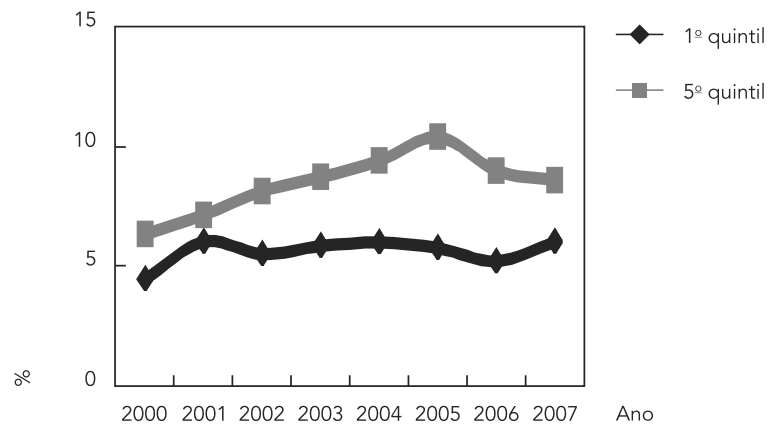

2i) Média da ação coletiva de escovação dental supervisionada (Pacto pela Saúde), segundo grupos de UF do 1ㅇ quintil e 5o quintil selecionadas pelo IDH de 2000. Brasil, 2008-2011

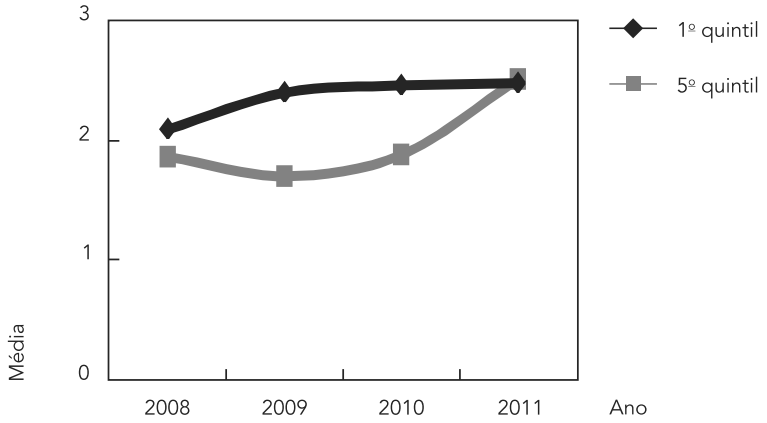

2h) Proporção de procedimentos odontológicos especializados em relação às ações odontológicas individuais (PIAB), segundo grupos de UF do 1ㅇ quintil e 5ㅇ quintil selecionadas pelo IDH de 2010. Brasil, 2000-2007 **

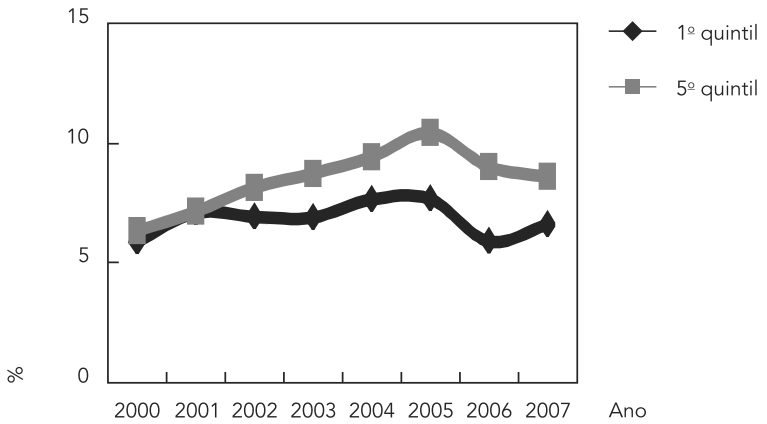

2j) Média da ação coletiva de escovação dental supervisionada (Pacto pela Saúde), segundo grupos de UF do 1o quintil e 5o quintil selecionadas pelo IDH de 2010. Brasil, 2008-2011

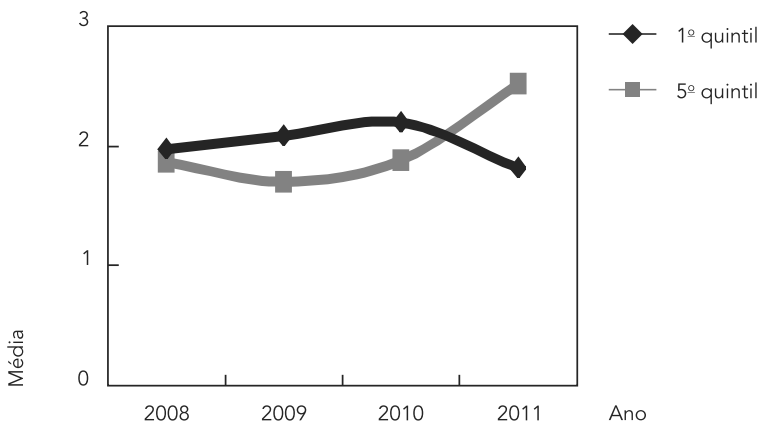

(continua)

A Cobertura populacional estimada das ESB da ESF também apresentou associação negativa com o IDH, aumentando com o tempo. Deve-se considerar, nesse caso, que apesar do incentivo para expansão das ESB ser relativamente novo dentro da ESF 43, já foi possível evidenciar diferenças na comparação entre os grupos, evidenciando que também as ESB estão sendo alocadas com maior demanda.

A Cobertura populacional estimada pelas ESB também manteve a tendência pró-equidade. Esse dado se deve à grande proporção de dentistas contratados pelo SUS nas regiões Norte e
Nordeste, atingindo porcentagens semelhantes às de médicos no serviço público. Nas regiões Sul e Sudeste, onde há mais dentistas trabalhando (respectivamente, $16 \%$ e $59 \%$ do total no país), foi relativamente menor a sua incorporação pelo serviço público. Esses dados indicam o esforço em propiciar maior provisão de atendimento odontológico público nas regiões onde são mais necessários 28,41 . 
Figura 2 (continuação)

Evolução dos indicadores de utilização, segundo grupos de Unidade da Federação (UF) com menor (1ㅇquintil) e maior (5 quintil) Índice de Desenvolvimento Humano (IDH).

2k) Média da ação coletiva de escovação dental supervisionada (Pacto pela Saúde/COAP), segundo grupos de UF do 1ㅇ quintil e 5ㅇ quintil selecionadas pelo IDH de 2000. Brasil, 2008-2012

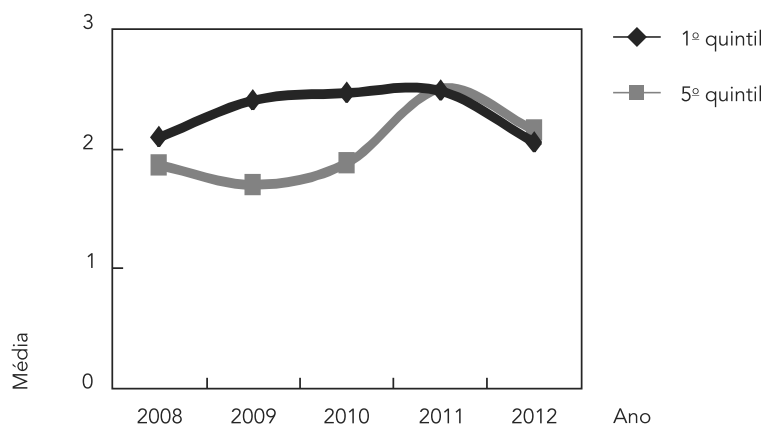

21) Média da ação coletiva de escovação dental supervisionada (Pacto pela Saúde/COAP), segundo grupos de UF do 1ㅇ quintil e 5으 quintil selecionadas pelo IDH de 2010. Brasil, 2008-2012

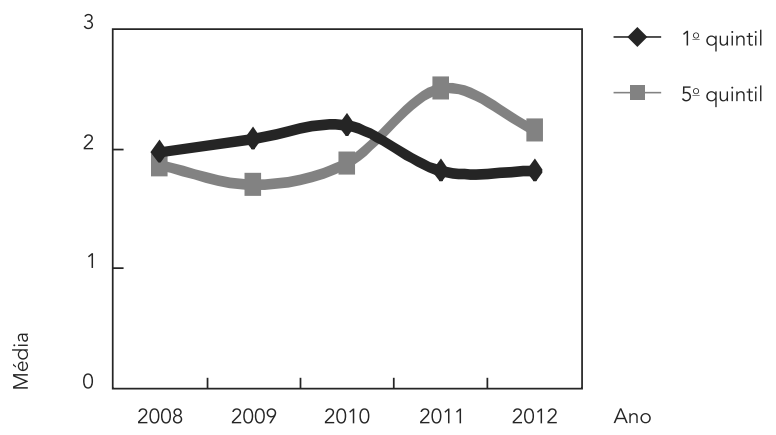

COAP: Contrato Organizativo da Ação Pública de Saúde; PIAB: Pacto de Indicadores da Atenção Básica.

* Diferença estatisticamente significante entre os quintis;

** Diferença estatisticamente significante entre os anos.

Tabela 3

Análise multivariada de regressão linear de efeitos mistos (modelagem intercepto randômico) entre indicadores de utilização e diferentes quintis do Índice de Desenvolvimento Humano (IDH).

\begin{tabular}{|c|c|c|c|c|c|c|c|c|}
\hline & \multicolumn{4}{|c|}{ IDH 2000} & \multicolumn{4}{|c|}{ IDH 2010} \\
\hline & $\beta$ & DP & IC95\% & $\begin{array}{c}\text { Valor de } \\
\qquad p\end{array}$ & $\beta$ & DP & IC95\% & $\begin{array}{c}\text { Valor de } \\
p\end{array}$ \\
\hline \multicolumn{9}{|c|}{ Cobertura de primeira consulta odontológica } \\
\hline \multicolumn{9}{|c|}{ programática (PIAB) } \\
\hline \multicolumn{9}{|l|}{ Efeito fixo } \\
\hline Intercepto & 16,96 & 2,05 & 12,$93 ; 20,99$ & $<0,001$ & 16,55 & 2,08 & 12,$46 ; 20,63$ & $<0,001$ \\
\hline Quintil & $-1,21$ & 0,56 & $-2,31 ;-0,10$ & 0,03 & $-1,13$ & 0,57 & $-2,25 ;-0,01$ & 0,04 \\
\hline Ano & $-0,13$ & 0,58 & $-1,29 ; 1,02$ & 0,81 & $-0,11$ & 0,58 & $-1,25 ; 1,04$ & 0,86 \\
\hline \multicolumn{9}{|l|}{ Efeito aleatório } \\
\hline Intercepto & & 3,08 & & & & 3,12 & & \\
\hline Resíduo & & 1,17 & & & & 1,17 & & \\
\hline \multicolumn{9}{|c|}{ Cobertura da ação coletiva escovação dental } \\
\hline \multicolumn{9}{|c|}{ supervisionada (PIAB) } \\
\hline \multicolumn{9}{|l|}{ Efeito fixo } \\
\hline Intercepto & 1,56 & 1,03 & $-0,46 ; 3,58$ & 0,13 & 1,43 & 1,05 & $-0,64 ; 3,51$ & 0,17 \\
\hline Quintil & 0,09 & 0,28 & $-0,46 ; 0,65$ & 0,74 & 0,12 & 0,29 & $-0,44 ; 0,69$ & 0,66 \\
\hline Ano & 0,05 & 0,17 & $-0,28 ; 0,39$ & 0,75 & 0,01 & 0,17 & $-0,33 ; 0,35$ & 0,94 \\
\hline \multicolumn{9}{|l|}{ Efeito aleatório } \\
\hline Intercepto & & 1,59 & & & & 1,63 & & \\
\hline Resíduo & & 0,34 & & & & 0,35 & & \\
\hline
\end{tabular}

(continua) 


\begin{tabular}{|c|c|c|c|c|c|c|c|c|}
\hline & \multicolumn{4}{|c|}{ IDH 2000} & \multicolumn{4}{|c|}{ IDH 2010} \\
\hline & $\beta$ & DP & IC95\% & $\begin{array}{c}\text { Valor de } \\
\mathrm{p}\end{array}$ & $\beta$ & DP & IC95\% & $\begin{array}{c}\text { Valor de } \\
p\end{array}$ \\
\hline \multicolumn{9}{|c|}{ Média de procedimentos odontológicos básicos } \\
\hline \multicolumn{9}{|l|}{ individuais (PIAB) } \\
\hline \multicolumn{9}{|l|}{ Efeito fixo } \\
\hline Intercepto & 0,52 & 0,09 & 0,$34 ; 0,70$ & $<0,001$ & 0,51 & 0,09 & 0,$34 ; 0,69$ & $<0,001$ \\
\hline Quintil & $-0,004$ & 0,02 & $-0,05 ; 0,04$ & 0,87 & $-0,001$ & 0,02 & $-0,04 ; 0,05$ & 0,97 \\
\hline Ano & 0,007 & 0,01 & 0,$0007 ; 0,01$ & 0,02 & 0,005 & 0,003 & $-0,001 ; 0,01$ & 0,15 \\
\hline \multicolumn{9}{|l|}{ Efeito aleatório } \\
\hline Intercepto & & 0,14 & & & & 0,13 & & \\
\hline Resíduo & & 0,06 & & & & 0,06 & & \\
\hline \multicolumn{9}{|c|}{ Proporção de procedimentos odontológicos } \\
\hline \multicolumn{9}{|c|}{ especializados em relação às ações odontológicas } \\
\hline \multicolumn{9}{|c|}{ individuais (PIAB) } \\
\hline \multicolumn{9}{|l|}{ Efeito fixo } \\
\hline Intercepto & 3,99 & 0,82 & 2,$38 ; 5,60$ & $<0,001$ & 5,56 & 1,69 & 2,$35 ; 8,98$ & 0,001 \\
\hline Quintil & 0,72 & 0,22 & 0,$28 ; 1,15$ & 0,001 & 0,41 & 0,46 & $-0,49 ; 1,32$ & 0,37 \\
\hline Ano & 0,24 & 0,06 & 0,$12 ; 0,35$ & $<0,001$ & 0,02 & 0,06 & 0,$07 ; 0,33$ & 0,002 \\
\hline \multicolumn{9}{|l|}{ Efeito aleatório } \\
\hline Intercepto & & 0,18 & & & & 2,59 & & \\
\hline Resíduo & & 1,07 & & & & 1,19 & & \\
\hline \multicolumn{9}{|c|}{ Média da ação coletiva de escovação dental } \\
\hline \multicolumn{9}{|c|}{ supervisionada (Pacto pela Saúde) } \\
\hline \multicolumn{9}{|c|}{ Efeito fixo } \\
\hline Intercepto & 2,85 & 1,17 & $-1,44 ; 3,15$ & 0,47 & 1,17 & 1,17 & $-1,13 ; 3,47$ & 0,31 \\
\hline Quintil & $-0,09$ & 0,21 & $-0,52 ; 0,33$ & 0,66 & $-0,006$ & 0,24 & $-0,48 ; 0,47$ & 0,98 \\
\hline Ano & 0,17 & 0,09 & $-0,01 ; 0,34$ & 0,06 & 0,09 & 0,08 & $-0,07 ; 0,24$ & 0,28 \\
\hline \multicolumn{9}{|l|}{ Efeito aleatório } \\
\hline Intercepto & & 1,18 & & & & 1,35 & & \\
\hline Resíduo & & 0,58 & & & & 0,52 & & \\
\hline \multicolumn{9}{|c|}{ Média da ação coletiva de escovação dental } \\
\hline \multicolumn{9}{|c|}{ supervisionada (Pacto pela Saúde/COAP) } \\
\hline \multicolumn{9}{|c|}{ Efeito fixo } \\
\hline Intercepto & 1,68 & 1,04 & $-0,35 ; 3,71$ & 0,12 & 1,55 & 1,07 & $-0,53 ; 3,65$ & 0,14 \\
\hline Quintil & $-0,07$ & 0,20 & $-0,46 ; 0,32$ & 0,73 & 0,01 & 0,23 & $-0,44 ; 0,46$ & 0,96 \\
\hline Ano & 0,07 & 0,07 & $-0,08 ; 0,21$ & 0,35 & 0,04 & 0,08 & $-0,09 ; 0,17$ & 0,55 \\
\hline \multicolumn{9}{|c|}{ Efeito aleatório } \\
\hline Intercepto & & 1,09 & & & & 1,27 & & \\
\hline Resíduo & & 0,66 & & & & 0,60 & & \\
\hline
\end{tabular}

B: coeficiente de regressão; COAP: Contrato Organizativo da Ação Pública de Saúde; DP: desvio padrão; IC95\%: intervalo de $95 \%$ de confiança; PIAB: Pacto de Indicadores da Atenção Básica.

\section{Indicadores de utilização}

Dois indicadores de saúde bucal relacionados com a utilização dos serviços estiveram associados ao IDH. A Cobertura de primeira consulta odontológica programática apresentou associação negativa com o quintil de IDH, ao passo que a Proporção de procedimentos odontológicos especializados em relação às ações odontológicas individuais registrou associação positiva. Porém, essa associação foi encontrada apenas nos grupos selecionados pelo IDH de 2000. Isso indica que o número de procedimentos especializados em relação aos demais procedimentos no Pará seja superior ao da Paraíba, como observado no DATASUS. Em 2000, esse indicador era 
$10,27 \%$ no Pará e 4,47\% na Paraíba, e no último ano disponível (2007) era 10,76\% no Pará e 8,36\% na Paraíba. É possível que a população paraense utilize preferencialmente os centros especializados devido à dificuldade de acesso às unidades básicas 44 .

Além disso, o fato de as diferenças terem sido percebidas apenas entre as UF selecionadas a partir do IDH de 2000, sugere que para a utilização dos procedimentos odontológicos especializados esteja havendo uma atenuação das diferenças entre as UF com maior e menor concentração de renda, graças à ampliação progressiva e direcionamento dos recursos públicos de atenção odontológica 41 .

A Cobertura de primeira consulta odontológica programática apresentou associação com o IDH no sentido pró-equidade. Possivelmente uma resposta ao aumento de incentivo em 2004 para as ESF e saúde bucal para os municípios com menor IDH. Paralelamente, a política nacional Brasil Sorridente, a partir de 2004, incorporou incentivos adicionais a esse programa, como insumos e equipamentos odontológicos, o que pode ter potencializado esse efeito 45 .

Quatro dos seis indicadores de utilização dos serviços de saúde bucal não diferiram entre as UF de maior e menor IDH. Dados semelhantes foram encontrados em estudos de nível individual no Brasil, nos quais não foi observada equidade na utilização dos serviços de saúde bucal 46,47,48. Maior utilização é registrada em pessoas com maior renda 49 , escolaridade $46,47,48$ e com melhor percepção de saúde bucal 46,47.

Esses dados discordam de estudos anteriores 6,19,29, em que a utilização dos serviços de saúde bucal foi considerada a favor da tendência de equidade. Essa divergência pode ser explicada pelas características das regiões Sul e Sudeste onde essas pesquisas foram realizadas. Historicamente, essas regiões são mais bem estruturadas político e socioeconomicamente, possibilitando que os municípios com menor IDH fossem favorecidos também em relação aos serviços odontológicos. Entretanto, no presente trabalho, as UF com o menor IDH coincidiram com os estados das regiões Norte e Nordeste, menos estruturados em relação à provisão dos serviços em saúde. Para esses estados, embora pareça haver maior oportunidade de acesso, o impacto das políticas públicas sobre a utilização dos serviços ainda não pôde ser percebida.

\section{Limites e potencialidades do estudo}

O estudo apresenta algumas restrições inerentes ao seu próprio desenho. Ao se optar por trabalhar com dados secundários, deve-se considerar a possibilidade de fragilidade dos registros e do controle de qualidade das informações coletadas. Além disso, a análise de dados agregados no nível de UF não leva em consideração o impacto das desigualdades sociais em níveis intraestadual e intramunicipal. Dessa forma, embora tenha sido observada associação negativa entre indicadores de acesso testados e o IDH, não se pode afirmar que a tendência de pró-equidade tenha ocorrido no nível individual, pois as validades dos dados ficam submetidas ao risco de falácia ecológica.

Inferências sobre a melhoria da situação de saúde bucal da população brasileira a partir da tendência pró-equidade observada devem ser feitas com cautela. Melhorias desses indicadores não implicam necessariamente mudanças no modelo assistencial 50, nem na saúde dos indivíduos 51,52.

Neste estudo, não foi considerado o Rol de Diretrizes, Objetivos, Metas e Indicadores 20132015 53. Isso porque dentre os indicadores de saúde bucal adotados nesta pactuação, dois Cobertura populacional estimada pelas ESB e Média da ação coletiva de escovação dental supervisionada - já haviam sido considerados nas pactuações Pacto pela Saúde 21 e transição Pacto pela Saúde/COAP 23 no mesmo período, 2008-2012, correspondendo aos dados validados mais atuais por ocasião da coleta. Já o indicador Proporção de exodontia em relação aos procedimentos, embora não tenha sido utilizado nas pactuações anteriores, verifica a redução do percentual de exodontias em relação aos procedimentos preventivos e curativos, portanto, indicando mudanças no modelo assistencial e não necessariamente a utilização ou acesso aos serviços, as duas perspectivas adotadas para este trabalho. Ademais, o indicador Cobertura de equipes da Atenção Básica também já foi considerado na transição Pacto pela Saúde/COAP 23.

A descontinuidade dos indicadores de saúde bucal nos sistemas de informação do SUS dificulta a comparação longitudinal dos dados. Na presente investigação, as séries históricas foram construídas por bloco de pactuação (PIAB 32, Pacto pela Saúde 21 e transição Pacto pela Saúde/ COAP 23), respeitando o intervalo de tempo de cada pacto e mantendo constante o cálculo do indicador, reduzindo o viés de aferição.

A estrutura hierárquica de dados longitudinais, em que medidas repetidas são aninhadas dentro da UF, gera dependência entre as observações feitas ano a ano e erros correlacionados. Essas suposições exigem modelagem da matriz de covariância dos dados, que não seria alcançada com análises convencionais de regressão linear. A regressão linear de efeitos mistos, 
adotada neste estudo, produz estimativas dos erros-padrão dos coeficientes do modelo com menor vício, na medida em que incorpora a estrutura de dependência dos dados nas estimativas de erro-padrão 54 .

Por fim, destaca-se o caráter pioneiro desta investigação, que avalia os indicadores de saúde bucal pactuados nos instrumentos de negociação mais recentes em todo o território nacional.

\section{Considerações finais}

Em conclusão, parece haver uma tendência próequidade em relação aos indicadores de opor- tunidade de acesso à Atenção Básica em saúde bucal, o que pode refletir os esforços das políticas públicas em diminuir as iniquidades em saúde. Porém, essa tendência ainda não se reflete nos indicadores de uso dos serviços, nos quais a proporção de procedimentos odontológicos especializados em relação às ações odontológicas individuais é maior entre as UF pertencentes ao quintil de maior IDH. Isso aponta para lacunas na garantia dos direitos previstos pelo SUS. Em última análise, estes achados podem contribuir para uma avaliação das políticas públicas voltadas para saúde bucal vigentes no Brasil, auxiliando gestores e pesquisadores no planejamento das ações e serviços de saúde.

\section{Colaboradores}

J. K. B. Fernandes e E. B. A. F. Thomaz conceberam o estudo, revisaram a literatura, realizaram as análises e interpretação dos dados, redigiram o manuscrito e aprovaram a versão final a ser publicada. J. R. O. Pinho revisou a literatura, realizou as análises e interpretação dos dados e redigiu o manuscrito. R. C. S. Queiroz contribuiu com a redação e aprovação da versão final a ser publicada.

\section{Agradecimentos}

À FAPEMA pelo apoio financeiro (Programa de Apoio à Publicação de Artigos, Edital no 013/2015). 


\section{Referências}

1. Sheiham A, Alexander D, Cohen L, Marinho V, Moysés $\mathrm{S}$, Petersen P, et al. Global oral health inequalities: task group - implementation and delivery of oral health strategies. Adv Dent Res 2011; 23:25967.

2. Szwarcwald CL, Bastos FI, Esteves MAP, Andrade CLT, Paez MS, Medici EV, et al. Desigualdade de renda e situação de saúde: o caso do Rio de Janeiro. Cad Saúde Pública 1999; 15:15-28.

3. Peres KG, Bastos JRM, Latorre MRDO. Severidade de cárie em crianças e relação com aspectos sociais e comportamentais. Rev Saúde Pública 2000; 34:402-8.

4. Irigoyen ME, Maupome, G, Mejía AM. Caries experience and treatment needs in a 6-to 12-year-old urban population in relation to socio-economic status. Community Dent Health 1999; 16:245-9.

5. Antunes JLF, Narvai PC, Nugent ZJ. Measuring inequalities in the distribution of dental caries. Community Dent Oral Epidemiol 2004; 32:41-8.

6. Baldani MH, Vasconcelos AGG, Antunes JLF. Associação do índice CPO-D com indicadores socioeconômicos e de provisão de serviços odontológicos no Estado do Paraná, Brasil. Cad Saúde Pública 2004; 20:143-52.

7. Paim J, Travassos C, Almeida C, Bahia L, Macinko J. The Brazilian health system: history, advances, and challenges. Lancet 2011;377:1778-97.

8. Victora CG, Barreto ML, Leal MC, Monteiro CA, Schmidt MI, Paim J, et al. Condições de saúde e inovações nas políticas de saúde no Brasil: o caminho a percorrer. Lancet 2011; 377:90-102.

9. Viana SM, Nunes A, Santos JRS, Barata RB. Medindo as desigualdades em saúde no Brasil: uma proposta de monitoramento. Brasília: Organização Pan-Americana da Saúde/Instituto de Pesquisa Econômica Aplicada; 2011.

10. Ministério da Saúde. Portaria no 2.203 , de 5 de novembro de 1996. Diário Oficial da União 1996; 6 nov.

11. Vazquez DA. Efeitos da regulação federal sobre o financiamento da saúde. Cad Saúde Pública 2011; 27:1201-12.

12. Machado ENM, Fortes FBCTP, Somarriba M. Efeitos da introdução do PAB sobre a distribuição de recursos e prestação de serviços: o caso de Minas Gerais. Ciênc Saúde Coletiva 2004; 9:99-111.

13. Melamed C, Costa NR. Inovações no financiamento federal à Atenção Básica. Ciênc Saúde Coletiva 2003; 8:393-401.

14. Souza RR. Redução das desigualdades regionais na alocação dos recursos federais para a saúde. Ciênc Saúde Coletiva 2003; 8:449-60.

15. Ministério da Saúde. Portaria no 3.925, de 13 de novembro de 1998. Diário Oficial da União 1998; 17 nov.

16. Ministério da Saúde. Portaria no 476, de 14 de abril de 1999. Diário Oficial da União 1999; 22 abr.

17. Departamento de Atenção Básica, Secretaria de Atenção à Saúde, Ministério da Saúde. Análise dos indicadores da política nacional de atenção básica no Brasil. Brasília: Ministério da Saúde; 2008.
18. Departamento de Atenção Básica, Secretaria de Atenção à Saúde, Ministério da Saúde. Instrumento de negociação qualificador do processo de gestão do SUS. Rev Bras Saúde Matern Infant 2003; 3:221-4.

19. Fischer TK, Peres KG, Kupek E, Peres MA. Indicadores de atenção básica em saúde bucal: associação com as condições socioeconômicas, provisão de serviços, fluoretação de águas e a estratégia de saúde da família no Sul do Brasil. Rev Bras Epidemiol 2010; 13:126-38.

20. Tamaki EM, Tanaka OY, Felisberto E, Alves CKA, Drumond Junior M, Bezerra LCA, et al. Metodologia de construção de um painel de indicadores para o monitoramento e a avaliação da gestão do SUS. Ciênc Saúde Coletiva 2012; 17:839-49.

21. Ministério da Saúde. Pacto pela saúde, 2015. http://portal.saude.gov.br/portal/saude/area. cfm?id_area=1021 (acessado em 14/Jan/2015).

22. Ministério da Saúde. Orientações acerca dos indicadores de monitoramento, avaliação do pacto pela saúde, nos componentes pela vida e de gestão para o biênio 2010-2011. Brasília: Ministério da Saúde; 2011.

23. Departamento de Articulação Interfederativa, Secretaria de Gestão Estratégica e Participativa, Ministério da Saúde. Guia para a elaboração do Contrato Organizativo da Ação Pública: construindo o COAP passo a passo. Brasília: Ministério da Saúde; 2014.

24. Barros AJD, Bertoldi AD. Desigualdades na utilização e no acesso a serviços odontológicos: uma avaliação em nível nacional. Ciênc Saúde Coletiva 2002; 7:709-17.

25. Departamento de Atenção Básica, Secretaria de Atenção à Saúde, Ministério da Saúde. Portaria no 2.488, de 21 de outubro de 2011. PNAB - Política Nacional de Atenção Básica. Brasília: Ministério da Saúde; 2011.

26. Departamento de Atenção Básica, Secretaria de Atenção à Saúde, Ministério da Saúde. Programa Nacional de Melhoria do Acesso e da Qualidade da Atenção Básica (PMAQ-AB), 2012. http://dab. saude.gov.br/sistemas/pmaq/faq.php (acessado em 03/Mai/2015).

27. Fausto MCR, Giovanella L, Mendonça MHM, Seidl H, Gagno J. A posição da Estratégia Saúde da Família na rede de atenção à saúde na perspectiva das equipes e usuários participantes do PMAQ-AB. Saúde Debate 2014; 38(Spe):13-33.

28. Peres KG, Peres MA, Boing AF, Bertoldi AD, Bastos JL, Barros AJD. Redução das desigualdades sociais na utilização de serviços odontológicos no Brasil entre 1998 e 2008. Rev Saúde Pública 2012; 46:250-8.

29. Baldani MH, Almeida ES, Antunes JLF. Serviços públicos odontológicos: provisão e equidade no Estado do Paraná, Brasil. Rev Saúde Pública 2009; 43:446-54.

30. Junqueira SR, Araújo ME, Antunes JLF, Narvai PC. Indicadores socioeconômicos e recursos odontológicos em municípios do Estado de São Paulo, Brasil, no final do Século XX. Epidemiol Serv Saúde 2006; 15:41-53. 
31. Programa das Nações Unidas para o Desenvolvimento. Atlas de Desenvolvimento Humano no Brasil. http://www.pnud.org.br/atlas/ (acessado em 14/Jan/2015).

32. Ministério da Saúde. Portaria no 493, de 10 de março de 2006. Diário Oficial da União 2006; 13 mar.

33. Ministério da Saúde. Manual do Sistema de Informação da Atenção Básica (SIAB). Brasília: Ministério da Saúde; 2000

34. Departamento de Informática do SUS. Pacto de Atenção Básica 2006. Notas técnicas. http:// tabnet.datasus.gov.br/cgi/pacto/2006/Nota_Tec nica_Indicadores.pdf (acessado em 14/Jan/2015).

35. Ministério da Saúde. Portaria no 3.840, de 7 de dezembro de 2010. Diário Oficial da União 2010; 10 Dez.

36. Departamento de Informática do SUS. Pacto pela Saúde 2010/2011: dados preliminares. Notas técnicas. http://tabnet.datasus.gov.br/cgi/pacto/2010/ Nota_Tecnica_Indicadores.pdf (acessado em 14/Jan/2015).

37. Departamento de Informática do SUS. Nota técnica dos indicadores de transição Pacto pela Saúde e COAP - 2012. http://tabnet.datasus.gov.br/ pacto/2012/Nota_Tecnica_Indicadores.pdf (acessado em 14/Jan/2015).

38. Viana ALA, Fausto MCR, Lima LD. Política de saúde e eqüidade. São Paulo Perspect 2003; 17:58-68.

39. Rabe-Hesketh, S, Skrondal A. Multilevel and longitudinal modeling using Stata. 2nd Ed. College Station: Stata Press; 2008.

40. Fitzmaurice GM, Ravichandran C. A primer in longitudinal data analysis. Circulation 2008; 118:2005-10.

41. Antunes JLF, Narvai PC. Políticas de saúde bucal no Brasil e seu impacto sobre as desigualdades em saúde. Rev Saúde Pública 2010; 44:360-5.

42. Conill EM. Ensaio histórico-conceitual sobre a Atenção Primária à Saúde: desafios para a organização de serviços básicos e da Estratégia Saúde da Família em centros urbanos no Brasil. Cad Saúde Pública 2008; 24 Suppl 1: S7-27.

43. Souza TMS, Roncalli AG. Saúde bucal no Programa Saúde da Família: uma avaliação do modelo assistencial. Cad Saúde Pública 2007; 23:2727-39.

44. Dias de Lima L, d'Ávila Viana AL, Machado CV, Vercesi de Albuquerque M, Oliveira RG, Iozzi FL, et al. Regionalization and access to healthcare in Brazilian states: historical and political-institutional conditioning factors. Ciênc Saúde Coletiva 2012; 17:2881-92.
45. Pucca Jr. GA. A política nacional de saúde bucal como demanda social. Ciênc Saúde Coletiva 2006; 11:243-6.

46. Gomes AMM, Thomaz EBAF, Alves MTSSB, Silva AAM, Silva RA. Fatores associados ao uso dos serviços de saúde bucal: estudo de base populacional em municípios do Maranhão, Brasil. Ciênc Saúde Coletiva 2014; 19:629-40.

47. Baldani MH, Brito WH, Lawder JAC, Mendes YBE, Silva FFM, Antunes JLF. Individual determinants of dental care utilization among low-income adult and elderly individuals. Rev Bras Epidemiol 2010; 13:150-62.

48. Barros AJD, Bertoldi AD. Desigualdades na utilização e no acesso a serviços odontológicos: uma avaliação em nível nacional. Ciênc Saúde Coletiva 2002; 7:709-17.

49. Gilbert GH, Duncan RP, Shelton BJ. Social determinants of tooth loss. Health Serv Res 2003; 38:1843-62.

50. Pereira CRS, Roncalli AG, Cangussu MCT, Noro LRA, Patrício AAR, Lima KC. Impact of the Family Health Strategy: an analysis in cities in Northeast Brazil with more than 100,000 inhabitants. Cad Saúde Pública 2012; 28:449-62.

51. Soares FF, Figueiredo CRL, Borges NCM, Jordão RA, Freire MCM. Oral health teamwork in brazilian family healthcare strategy: an analysis of studies published between 2001 and 2008. Ciênc Saúde Coletiva 2011; 16:3169-80.

52. Nascimento AC, Moysés ST, Werneck RI, Moysés SJ. Oral health in the context of primary care in Brazil. Int Dent J 2013; 63:237-43.

53. Ministério da Saúde. Caderno de diretrizes, objetivos, metas e indicadores 2013-2015. Brasília: Ministério da Saúde; 2013. (Série Articulação Interfederativa, volume 1).

54. Fausto MA, Carneiro M, Antunes CMF, Pinto JA, Colosimo EA. O modelo de regressão linear misto para dados longitudinais: uma aplicação na análise de dados antropométricos desbalanceados. Cad Saúde Pública 2008; 24:513-24. 


\section{Abstract}

This study tested the hypothesis that oral health indicators from the Pact for Primary Healthcare 2006, the Biennial Pact for Health 2010-2011, and the indicators for the transition from the Pact for Health to the 2012 Organizational Contract for Public Action in Health did not differ between states of Brazil with different Human Development Indices (HDI). A longitudinal ecological study was performed, comparing the states of Brazil with the highest and lowest HDIs. Data were obtained from the information systems of the Brazilian Unified National Health System (SUS) and the United Nations Development Program (UNDP) and submitted to mixed-effects linear regression (alpha $=$ 5\%). All the indicators for opportunity of access to oral health care were inversely associated with HDI. For in dicators of use, the association only occurred with two indicators. The results showed a trend towards equity for indicators of opportunity of access to oral health.

Health Inequalities; Social Inequity; Health

Status Indicators; Oral Health

\section{Resumen}

Fue evaluada la hipótesis de que los indicadores de salud bucal obtenidos en el Pacto de Atención Básica de 2006, en el Pacto de Salud del bienio 2010/2011 e indicadores de transición entre el Pacto por la Salud y Contrato Organizativo de Acción Pública en Salud de 2012, no fueron diferentes entre las Unidades Federativas (UF) brasileñas con diferentes Índices de Desarrollo Humano (IDH). Para eso, se realizó un estudio longitudinal ecológico comparando las UF de Brasil con extremos del IDH. Los datos fueron obtenidos de los sistemas de información del Sistema Único de Salud (SUS) y del Programa de Naciones Unidas para el Desarrollo, y sometidos a un análisis de regresión lineal de efectos mixtos (alfa $=5 \%$ ). Todos los indicadores de oportunidad de acceso fueron asociados negativamente con el IDH. Para los de utilización, la asociación se produjo en apenas dos indicadores. Los resultados mostraron una tendencia evolutiva pro-equidad para los indicadores de oportunidad de acceso en relación con la salud bucal.

Desigualdades en la Salud; Inequidad Social; Indicadores de Salud; Salud Bucal
Recebido em 12/Fev/2015

Versão final reapresentada em 29/Mai/2015 Aprovado em 08/Jun/2015 